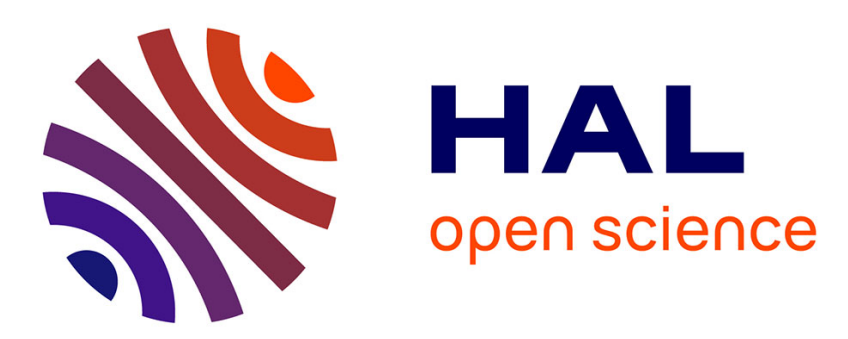

\title{
Theory of the spontaneous optical emission of nanosize photonic and plasmon resonators
}

Christophe Sauvan, Jean-Paul Hugonin, Ivan Maksymov, Philippe Lalanne

\section{To cite this version:}

Christophe Sauvan, Jean-Paul Hugonin, Ivan Maksymov, Philippe Lalanne. Theory of the spontaneous optical emission of nanosize photonic and plasmon resonators. Physical Review Letters, 2013, 110, pp.237401. 10.1103/PhysRevLett.110.237401 . hal-00850459

\section{HAL Id: hal-00850459 \\ https://hal-iogs.archives-ouvertes.fr/hal-00850459}

Submitted on 18 Nov 2015

HAL is a multi-disciplinary open access archive for the deposit and dissemination of scientific research documents, whether they are published or not. The documents may come from teaching and research institutions in France or abroad, or from public or private research centers.
L'archive ouverte pluridisciplinaire HAL, est destinée au dépôt et à la diffusion de documents scientifiques de niveau recherche, publiés ou non, émanant des établissements d'enseignement et de recherche français ou étrangers, des laboratoires publics ou privés. 


\title{
Theory of the Spontaneous Optical Emission of Nanosize Photonic and Plasmon Resonators
}

\author{
C. Sauvan, ${ }^{1, *}$ J. P. Hugonin, ${ }^{2}$ I. S. Maksymov, ${ }^{2}$ and P. Lalanne ${ }^{1, \dagger}$ \\ ${ }^{1}$ Laboratoire Photonique Numérique et Nanosciences, Institut d'Optique, Université Bordeaux, \\ CNRS, 33405 Talence, France \\ ${ }^{2}$ Laboratoire Charles Fabry, Institut d'Optique, CNRS, Université Paris Sud, \\ 2 avenue Augustin Fresnel, 91127 Palaiseau, France \\ (Received 13 November 2012; published 5 June 2013)
}

\begin{abstract}
We provide a self-consistent electromagnetic theory of the coupling between dipole emitters and dissipative nanoresonators. The theory that relies on the concept of quasinormal modes with complex frequencies provides an accurate closed-form expression for the electromagnetic local density of states of any photonic or plasmonic resonator with strong radiation leakage, absorption, and material dispersion. It represents a powerful tool to calculate and conceptualize the electromagnetic response of systems that are governed by a small number of resonance modes. We use the formalism to revisit Purcell's factor. The new formula substantially differs from the usual one; in particular, it predicts that a spectral detuning between the emitter and the resonance does not necessarily result in a Lorentzian response in the presence of dissipation. Comparisons with fully vectorial numerical calculations for plasmonic nanoresonators made of gold nanorods evidence the high accuracy of the predictions achieved by our semianalytical treatment.
\end{abstract}

The modification of the spontaneous decay $(\mathrm{SD})$ rate of a quantum emitter induced by an electromagnetic resonance, the so-called Purcell effect [1], is one of the very fundamental effects in quantum electrodynamics. With the advent of nanotechnologies, this effect is nowadays revisited at deep-subwavelength scales with new applications in nano-optical spectroscopy [2-5], nanolasers [6], coherent generation of plasmons [7-9], or broadband single-photon sources [10,11]. In his landmark note [1], Purcell introduced two important quantities, the quality factor $Q$ and the mode volume $V$, to quantify the maximum SD acceleration that may be achieved by coupling an emitter with a cavity in the weak-coupling regime $F=3 /\left(4 \pi^{2}\right)\left(\lambda_{0} / n\right)^{3} Q / V$, with $\lambda_{0} / n$ the resonance wavelength in the material surrounding the emitter.

The Purcell factor $F$ represents the maximum acceleration for an ideal coupling between the emitter and the cavity mode, i.e., a perfect spectral, spatial, and polarization matching. Once the mode field distribution is known, any deviation from perfect coupling can be calculated analytically. For instance, a spectral mismatch between the dipole frequency $\omega$ and the cavity resonance $\omega_{0}$ reduces the decay rate $\Gamma$ according to the usual Lorentzian line shape [12]

$$
\frac{\Gamma}{\Gamma_{0}}=F \frac{\omega_{0}^{2}}{\omega^{2}} \frac{\omega_{0}^{2}}{\omega_{0}^{2}+4 Q^{2}\left(\omega-\omega_{0}\right)^{2}},
$$

with $\Gamma_{0}$ the decay rate in the bulk material. The volume initially introduced by Purcell was a geometrical volume representing the spatial extent of the (microwave) resonator, but with the large amount of work devoted to optical microcavities in the 1990s, the mode volume definition has evolved to the usual expression $[12,13]$

$$
V=\frac{1}{\epsilon_{0} n^{2}} \int \epsilon(\mathbf{r})|\mathbf{E}(\mathbf{r})|^{2} d^{3} \mathbf{r}
$$

where $\epsilon_{0}$ is the vacuum permittivity, $\epsilon(\mathbf{r})$ is the permittivity of the resonator, and $\mathbf{E}$ is the cavity mode normalized such that its norm is unity at the antinode of the electric field. Although the mode volume is a purely electromagnetic quantity, its definition lacks a precise argument. Actually, $V$ is difficult to define for dissipative (non-Hermitian) systems, even for dielectric cavities where the energy dissipation simply arises from radiative leakage [13-15]. This theoretical difficulty has recently been underlined (without being solved) in the literature on metallic nanoresonators, for which absorption and dispersion have to be handled in addition to radiative leakage [16-18].

In this work, we abandon the usual description based on the electromagnetic energy in lossless and nondispersive media. From first-principles calculations based on Maxwell's equations and Fermi's golden rule, we propose a self-consistent classical theory for the emitter-cavity coupling. We derive a closed-form expression for the local density of states (LDOS) and a generalized Purcell formula that are valid for any nanocavity with radiative leakage, absorption, and material dispersion, including the important case of plasmonic nanoantennas [3-9,19]. In particular, we show that a cavity mode may decelerate the total $\mathrm{SD}$, even when it is spectrally and spatially matched with the emitter. We also evidence that a spectral mismatch does not necessarily result in a Lorentzian line shape as in Eq. (1). In fact, Eqs. (1) and (2) appear as a specific case of the present theory, valid in the limit $Q \rightarrow \infty$, i.e., when leakage, absorption, and thus dispersion can be neglected. Our theory is carefully validated by comparison with fully 
(a)

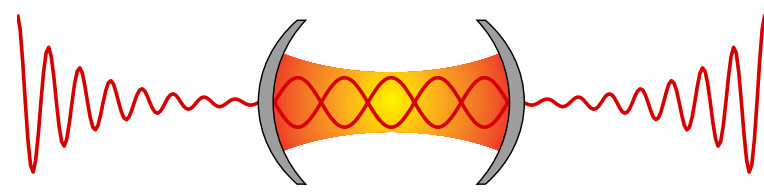

(b)

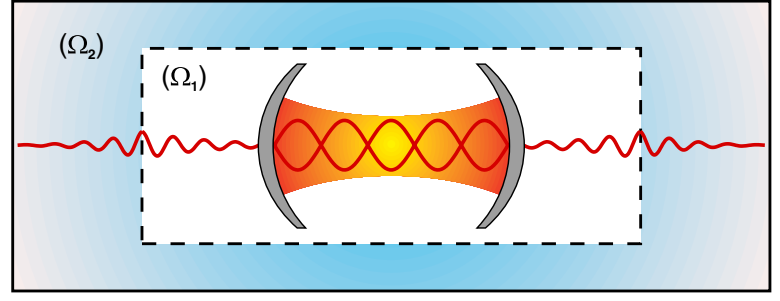

FIG. 1 (color online). The quasinormal mode (QNM) of an open cavity. (a) QNMs are electromagnetic field distributions that satisfy Maxwell's equations for a complex frequency. The field is stationary inside the resonator and exponentially diverging outside. Because of the divergence, QNMs are not compatible with the usual expression of the mode volume in Eq. (2), which relies on the electromagnetic energy. (b) QNM bounded by a perfectly matched-layer (PML) region shown with the bluish rectangular area $\left(\Omega_{2}\right)$. The latter has two crucial impacts. It allows us to suppress the divergence while preserving outgoing wave boundary conditions and to calculate the mode volume by integrating over the whole domain $\left(\Omega_{1}\right) \cup\left(\Omega_{2}\right)$ [23].

vectorial numerical results obtained for plasmonic nanoantennas made of gold nanorods.

It is instructive to first examine what are the issues behind the definition of $V$ for dissipative cavities. The cavity modes are the electromagnetic field distributions $\left(\tilde{\mathbf{E}}_{m}, \tilde{\mathbf{H}}_{m}\right)$ that are solutions of Maxwell's equations in the absence of a source $\nabla \times \tilde{\mathbf{E}}_{m}=i \tilde{\boldsymbol{\omega}}_{m} \mu \tilde{\mathbf{H}}_{m}$ and $\nabla \times \tilde{\mathbf{H}}_{m}=-i \tilde{\omega}_{m} \epsilon(\mathbf{r}) \tilde{\mathbf{E}}_{m}$ and that satisfy outgoing wave boundary conditions (the Sommerfeld radiation condition as $|\mathbf{r}| \rightarrow \infty$ ). The tilde is related to modal quantities hereafter. Because the energy leaks out or is absorbed, the modes possess a finite lifetime $\tau_{m}$. The eigenfrequency $\tilde{\omega}_{m}$ is thus complex with $\operatorname{Im}\left(\tilde{\omega}_{m}\right)=-1 / \tau_{m}$. Consistently with the literature on open systems for which the timeevolution operator is not Hermitian [13,14], we refer to these eigenmodes as quasinormal modes (QNMs), rather than normal modes, to emphasize that they are modes of a nonconservative system.

The definition of the quality factor does not raise any issue $Q=-\operatorname{Re}\left(\tilde{\omega}_{m}\right) /\left(2 \operatorname{Im}\left(\tilde{\omega}_{m}\right)\right)$, an expression that is related to energy balance arguments [20]. However, a problem arises with the definition of $V$. Actually, as $\operatorname{Im}\left(\tilde{\omega}_{m}\right)<0$, electromagnetic fields are amplified as they propagate (wave vectors become complex with a negative imaginary part). Thus, the QNM field diverges exponentially as $|\mathbf{r}| \rightarrow \infty$ [see Fig. 1(a)], and the volume integral in Eq. (2) is also exponentially diverging. This problem has been well known since the early studies on optical microcavities [12-15]. It has been bypassed by calculating $V$ for empirically related field distributions defined by Born-von
Kármán periodic boundary conditions $[12,14]$. For high- $Q$ cavities, the field that leaks in the clads is small compared to the field inside the cavity, and the difference between the volumes of the empiric "mode" and of the actual QNM is negligible $[12,15]$. For plasmonic nanocavities whose $Q$ 's rarely exceed a few tens, the definition of $V$ is even more critical, as was recently underlined $[17,18]$. Not only does the trick used for high- $Q$ dielectric cavities become largely unsubstantiated, but in addition, material dispersion and Ohmic losses have to be correctly handled. The lack of a sound theoretical framework is all the more detrimental, as the coupling between a quantum emitter and a plasmonic resonance is at the heart of important new paradigms, such as the stimulated generation of plasmons in volumes much smaller than the wavelength [7-9] or the loss compensation in metamaterials [21].

We consider a single emitter located at $\mathbf{r}=\mathbf{r}_{0}$ in the vicinity of absorptive, dispersive, and anisotropic nanostructures with open boundaries. The system is characterized by the position- and frequency-dependent permittivity and permeability tensors $\epsilon(\mathbf{r}, \omega)$ and $\mu(\mathbf{r}, \omega)$. We assume that the materials are reciprocal $\epsilon=\epsilon^{T}$ and $\mu=\mu^{T}$, where the superscript denotes matrix transposition. In the weak-coupling regime, the SD rate $\Gamma$ can be derived from Fermi's golden rule and the electric Green tensor [22]. For an electric-dipole transition at the frequency $\omega$ with a dipole moment $\mathbf{p}, \Gamma$ takes the form

$$
\Gamma=\frac{2}{\hbar} \operatorname{Im}\left[\mathbf{p}^{*} \cdot \mathbf{E}\left(\mathbf{r}_{0}\right)\right]
$$

where $\mathbf{E}$ is the total electric field that satisfies Maxwell's equations in the presence of the dipole, $\nabla \times \mathbf{E}=$ $i \omega \mu(\mathbf{r}, \omega) \mathbf{H}$, and $\nabla \times \mathbf{H}=-i \omega \epsilon(\mathbf{r}, \omega) \mathbf{E}-i \omega \mathbf{p} \delta\left(\mathbf{r}-\mathbf{r}_{0}\right)$, with $\delta$ the Dirac distribution.

We now make the sole assumption of this work by considering that the electromagnetic field $(\mathbf{E}, \mathbf{H})$ radiated by the dipole can be expanded onto a small set of QNMs

$$
\mathbf{E}(\mathbf{r}, \omega) \approx \sum_{m=1}^{M} \alpha_{m}(\omega) \tilde{\mathbf{E}}_{m}(\mathbf{r})
$$

where $\alpha_{m}$ are complex coefficients to be determined. A similar expression with the same $\alpha_{m}$ 's holds for the magnetic field. The number of QNMs is determined by increasing $M$ until convergence is reached $(M=1$ in Fig. 2, and $M=2$ in Fig. 3). If the system supports degenerate modes, they all have to be included in the expansion. The validity of Eq. (4) is questionable only when the expansion onto a small set of discrete modes neglects important decay channels [17]. For instance, an emitter located outside the cavity in the evanescent field is only weakly coupled to the resonance and mainly decays in the free-space continuum [23]. Hereafter, we first show that the $\alpha_{m}$ 's can easily be calculated without any approximation by solving a linear system. In a second step, we provide an approximate analytical expression for $\alpha_{m}$ from 
which we define a generalized Purcell formula for absorptive and dispersive nanocavities.

By applying the unconjugated form of Lorentz reciprocity [23] to the total field $(\mathbf{E}, \mathbf{H})$ created by the dipole at the frequency $\omega$ and to the $n$th mode $\left(\tilde{\mathbf{E}}_{n}, \tilde{\mathbf{H}}_{n}\right)$, we obtain $\int\left\{\mathbf{E} \cdot\left[\omega \boldsymbol{\epsilon}(\omega)-\tilde{\omega}_{n} \boldsymbol{\epsilon}\left(\tilde{\omega}_{n}\right)\right] \tilde{\mathbf{E}}_{n}-\mathbf{H} \cdot[\omega \mu(\omega)-\right.$ $\left.\left.\tilde{\omega}_{n} \mu\left(\tilde{\omega}_{n}\right)\right] \tilde{\mathbf{H}}_{n}\right\} d^{3} \mathbf{r}=-\omega \mathbf{p} \cdot \tilde{\mathbf{E}}_{n}\left(\mathbf{r}_{0}\right)$. We then use the modal expansion of Eq. (4) and obtain a linear system of $M$ equations $\sum_{m} B_{n m}(\omega) \alpha_{m}(\omega)=-\omega \mathbf{p} \cdot \tilde{\mathbf{E}}_{n}\left(\mathbf{r}_{0}\right)$, where the unknowns are the $\alpha_{m}$ 's and $B_{n m}(\omega)=\int\left\{\tilde{\mathbf{E}}_{m} \cdot[\omega \epsilon(\omega)-\right.$ $\left.\left.\tilde{\omega}_{n} \epsilon\left(\tilde{\omega}_{n}\right)\right] \tilde{\mathbf{E}}_{n}-\tilde{\mathbf{H}}_{m} \cdot\left[\omega \mu(\omega)-\tilde{\omega}_{n} \mu\left(\tilde{\omega}_{n}\right)\right] \tilde{\mathbf{H}}_{n}\right\} d^{3} \mathbf{r}$.

Let us first examine these equations for nondispersive materials. In this specific case, $\epsilon$ and $\mu$ are frequency independent and the coefficients $B_{n m}$ become $B_{n m}(\omega)=$ $\left(\omega-\tilde{\omega}_{n}\right) \int\left(\tilde{\mathbf{E}}_{m} \cdot \epsilon \tilde{\mathbf{E}}_{n}-\tilde{\mathbf{H}}_{m} \cdot \mu \tilde{\mathbf{H}}_{n}\right) d^{3} \mathbf{r}$. By using the orthogonality of the QNMs of nondispersive systems [23], we get $B_{n m}(\omega)=0$ for $n \neq m$ and $B_{n n}(\omega)=\left(\omega-\tilde{\omega}_{n}\right) \times$ $\int\left(\tilde{\mathbf{E}}_{n} \cdot \epsilon \tilde{\mathbf{E}}_{n}-\tilde{\mathbf{H}}_{n} \cdot \mu \tilde{\mathbf{H}}_{n}\right) d^{3} \mathbf{r}$. Therefore, in the absence of dispersion, the linear system of equations is diagonal and we trivially obtain $\alpha_{n}(\omega)=-\omega \mathbf{p} \cdot \tilde{\mathbf{E}}_{n}\left(\mathbf{r}_{0}\right) /\left[\left(\omega-\tilde{\boldsymbol{\omega}}_{n}\right) \times\right.$ $\left.\int\left(\tilde{\mathbf{E}}_{n} \cdot \epsilon \tilde{\mathbf{E}}_{n}-\tilde{\mathbf{H}}_{n} \cdot \mu \tilde{\mathbf{H}}_{n}\right) d^{3} \mathbf{r}\right]$. Note that $\alpha_{n}$ has a pole for $\omega=\tilde{\omega}_{n}$; the system resonates whenever the exciting frequency $\omega$ is close to one of the eigenfrequencies $\tilde{\omega}_{n}$.

In the general case of dispersive media, the QNMs are not orthogonal and the off-diagonal coefficients $B_{n m}(\omega)$ are not equal to zero. One thus has to solve a small linear system of $M$ equations. To guarantee a safe and accurate numerical implementation, we note that $B_{n m}(\omega)$ is null for $\omega=\tilde{\omega}_{m}$ and for any $n$ or $m$ as shown in [23], and we write $B_{n m}(\omega)=\left(\omega-\tilde{\omega}_{m}\right) A_{n m}(\omega)$, with $A_{n m}\left(\tilde{\omega}_{m}\right) \neq 0$. The linear system of equations can thus be rewritten as

$$
\sum_{m} A_{n m}(\omega) x_{m}(\omega)=-\omega \mathbf{p} \cdot \tilde{\mathbf{E}}_{n}\left(\mathbf{r}_{0}\right),
$$

where the unknowns are now $x_{m}(\omega)=\left(\omega-\tilde{\omega}_{m}\right) \alpha_{m}(\omega)$ and the coefficients $A_{n m}(\omega)$ are given by

$$
\begin{aligned}
A_{n m}(\omega)= & \frac{1}{\omega-\tilde{\omega}_{m}} \int\left\{\tilde{\mathbf{E}}_{m} \cdot\left[\omega \epsilon(\omega)-\tilde{\omega}_{n} \epsilon\left(\tilde{\omega}_{n}\right)\right] \tilde{\mathbf{E}}_{n}\right. \\
& \left.-\tilde{\mathbf{H}}_{m} \cdot\left[\omega \mu(\omega)-\tilde{\omega}_{n} \mu\left(\tilde{\omega}_{n}\right)\right] \tilde{\mathbf{H}}_{n}\right\} d^{3} \mathbf{r},
\end{aligned}
$$

with $A_{n m}\left(\tilde{\omega}_{n}\right)=0 \quad$ for $n \neq m$ and $A_{n n}\left(\tilde{\omega}_{n}\right)=$ $\int\left[\tilde{\mathbf{E}}_{n} \cdot(\partial(\omega \epsilon) / \partial \omega) \tilde{\mathbf{E}}_{n}-\tilde{\mathbf{H}}_{n} \cdot(\partial(\omega \mu) / \partial \omega) \tilde{\mathbf{H}}_{n}\right] d^{3} \mathbf{r}$. In this form, the system of equations is not singular and it can be easily solved for the $x_{n}$ 's. We get around the difficulty associated with the divergence of the QNMs by considering the field calculated in perfectly matched layers surrounding the resonator (see Fig. 1 and [23]). Then, the total decay rate is obtained from Eqs. (3) and (4), $\Gamma=(2 / \hbar) \operatorname{Im}\left[\sum_{m} \alpha_{m} \mathbf{p}^{*} \cdot \tilde{\mathbf{E}}_{m}\left(\mathbf{r}_{0}\right)\right]$. Equations (5) and (6) constitute the major result of this work, together with the approach developed for calculating the $A_{n m}$ 's [23]; they form an accurate and efficient tool to calculate the SD rate of an emitter placed in any complex nanocavity.
For dispersive materials, the linear system is not diagonal, and it is not possible in general to derive a closed-form expression for the SD rate. However, we can still show that $\alpha_{n}(\omega)$ has a pole and use this property to derive an approximate analytical expression. For $\omega=\tilde{\omega}_{n}$, since $A_{n m}\left(\tilde{\omega}_{n}\right)=0$ for $n \neq m$, the $n$th line of the system in Eq. (5) simply becomes $A_{n n}\left(\tilde{\omega}_{n}\right) x_{n}\left(\tilde{\omega}_{n}\right)=-\tilde{\omega}_{n} \mathbf{p} \cdot \tilde{\mathbf{E}}_{n}\left(\mathbf{r}_{0}\right)$. Since $x_{n}(\omega)=\left(\omega-\tilde{\omega}_{n}\right) \alpha_{n}(\omega)$, we obtain that $\alpha_{n}(\omega)$ has a pole for $\omega=\tilde{\omega}_{n}$, whose residue is given by $-\tilde{\omega}_{n} \mathbf{p} \cdot \tilde{\mathbf{E}}_{n}\left(\mathbf{r}_{0}\right) / A_{n n}\left(\tilde{\omega}_{n}\right)$. Therefore, we can write

$$
\begin{aligned}
\alpha_{n}(\omega)= & \frac{-\omega \mathbf{p} \cdot \tilde{\mathbf{E}}_{n}\left(\mathbf{r}_{0}\right)}{\left(\omega-\tilde{\omega}_{n}\right) \int\left[\tilde{\mathbf{E}}_{n} \cdot \frac{\partial(\omega \epsilon)}{\partial \omega} \tilde{\mathbf{E}}_{n}-\tilde{\mathbf{H}}_{n} \cdot \frac{\partial(\omega \mu)}{\partial \omega} \tilde{\mathbf{H}}_{n}\right] d^{3} \mathbf{r}} \\
& +f_{n}(\omega),
\end{aligned}
$$

where $f_{n}(\omega)$ is a nonresonant background that is negligible for $\omega \approx \tilde{\omega}_{n}$. We thus obtain an approximate closed-form expression for $\alpha_{n}$ valid in the vicinity of $\tilde{\omega}_{n}$.

For a dipole essentially coupled to a single resonance $\tilde{\mathbf{E}}$ (an important case in practice $[3,4,19]$ ), the SD rate is $\Gamma=(2 / \hbar) \operatorname{Im}\left[\alpha \mathbf{p}^{*} \cdot \tilde{\mathbf{E}}\left(\mathbf{r}_{0}\right)\right]$. Simple derivations lead to

$$
\frac{\Gamma}{\Gamma_{0}}=F \frac{\omega_{0}^{2}}{\omega^{2}} \frac{\omega_{0}^{2}}{\omega_{0}^{2}+4 Q^{2}\left(\omega-\omega_{0}\right)^{2}}\left[1+2 Q \frac{\omega-\omega_{0}}{\omega_{0}} \frac{\operatorname{Im}(V)}{\operatorname{Re}(V)}\right],
$$

with $\omega_{0}=\operatorname{Re}(\tilde{\omega})$ and $\Gamma_{0}=\omega^{3}|p|^{2} n /\left(3 \pi \epsilon_{0} \hbar c^{3}\right)$ the SD rate in a bulk material with a refractive index $n$. In Eq. (8), $F$ and $V$ are the generalized Purcell factor and mode volume

$$
\begin{gathered}
V=\frac{\int\left[\tilde{\mathbf{E}} \cdot \frac{\partial(\omega \epsilon)}{\partial \omega} \tilde{\mathbf{E}}-\tilde{\mathbf{H}} \cdot \frac{\partial(\omega \mu)}{\partial \omega} \tilde{\mathbf{H}}\right] d^{3} \mathbf{r}}{2 \epsilon_{0} n^{2}\left[\tilde{\mathbf{E}}\left(\mathbf{r}_{0}\right) \cdot \mathbf{u}\right]^{2}}, \\
F=\frac{3}{4 \pi^{2}}\left(\frac{\lambda_{0}}{n}\right)^{3} \operatorname{Re}\left(\frac{Q}{V}\right) .
\end{gathered}
$$

Note that we have considered a linearly polarized dipole $\mathbf{p}=p \mathbf{u}$, with $\mathbf{u}$ a unit vector. The volume integral in Eq. (9) extends over the whole domain $\left(\Omega_{1}\right) \cup\left(\Omega_{2}\right)$ and does not diverge despite the QNM divergence; see Fig. 1 and [23]. The generalized Purcell factor in Eq. (10) takes exactly the same form as the usual factor introduced by Purcell, except that $V$ is now a complex quantity, whose real and imaginary parts impact the SD rate on and off resonance. The mode volume in Eq. (9) explicitly considers material dispersion, as evidenced by the derivatives $\partial(\omega \epsilon) / \partial \omega$ and $\partial(\omega \mu) / \partial \omega$ taken at the complex frequency $\tilde{\omega}$. We emphasize that Eqs. (8)-(10) are valid for any dissipative system, even with large absorption.

For a conservative (closed and lossless) cavity, the QNM field is real, and Eqs. (8)-(10) reduce to the usual expressions, which then appear as valid in the limit of large $Q$ 's. Energy dissipation results in the appearance of an imaginary part in the QNM field, and thus in $V$. A comparison between Eqs. (1) and (8) evidences the stringent difference 
between a conservative and a dissipative (open and/or lossy) system: in general, the SD rate of spectrally detuned dipoles is not described by a Lorentzian line shape, especially if $\operatorname{Im}(V) / \operatorname{Re}(V) \geq 1 / Q$.

The present formalism is not only capable of accurately predicting the total decay rate but also the radiative $\left(\Gamma_{R}\right)$ and nonradiative $\left(\Gamma_{\mathrm{NR}}\right)$ decays. $\Gamma_{\mathrm{NR}}$ is due to absorption and is predicted by integrating the intensity over the lossy region

$$
\Gamma_{\mathrm{NR}}=\frac{2}{\hbar}|\alpha(\omega)|^{2} \int \operatorname{Im}(\epsilon)|\tilde{\mathbf{E}}(\mathbf{r})|^{2} d^{3} \mathbf{r},
$$

and the radiative decay rate is simply obtained by energy conservation $\Gamma_{\mathrm{R}}=\Gamma-\Gamma_{\mathrm{NR}}$.

We validate the present theory by carefully testing its predictions against fully vectorial calculations for plasmonic nanoantennas made of metallic nanorods. We first consider a single gold nanorod (see Fig. 2), which has received considerable attention to control the spontaneous emission $[3,4,19,24]$. In the spectral range of interest, a single QNM is dominant, namely, the dipolelike mode of the nanorod shown in Fig. 2(a). We have used this mode in Eqs. (8) and (11) to calculate the total and nonradiative decay rates for an on-axis dipole oriented parallel to the nanorod and located at a distance $d=$ $10 \mathrm{~nm}$ from the metal surface. As shown in Fig. 2(b), the predictions of our theory (solid curves) are in excellent agreement with fully vectorial numerical data (circles and squares) calculated with the aperiodic Fourier modal method [25] implemented in cylindrical coordinates [26]. (a)

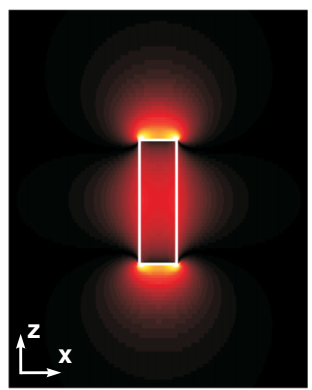

(b)

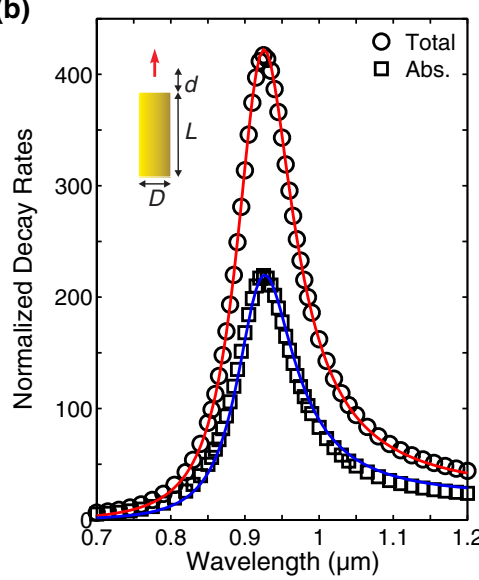

FIG. 2 (color online). Single metallic nanorod. A gold cylinder (diameter $D=30 \mathrm{~nm}$, length $L=100 \mathrm{~nm}$ ) is embedded in a host medium of refractive index $n=1.5$. (a) Electric field distribution $\left|\tilde{E}_{z}\right|$ of the dipolelike QNM with a complex frequency $2 \pi c / \tilde{\omega}=920+47 i \mathrm{~nm}$. (b) Normalized decay-rate spectrum for an on-axis dipole oriented parallel to the nanorod (red arrow) and located at $d=10 \mathrm{~nm}$. The circles and squares are fully vectorial data for the total $(\Gamma)$ and nonradiative $\left(\Gamma_{\mathrm{NR}}\right)$ decay rates. The solid curves are obtained from Eqs. (8) and (11) for the QNM shown in (a).
To calculate QNMs, one needs an analytical continuation of the gold permittivity for complex frequencies. We have used a Drude model that fits the tabulated data in [27], $\epsilon=1-\omega_{p}^{2} /\left(\omega^{2}+i \omega \gamma\right)$ with $\omega_{p}=1.26 \times$ $10^{16} \mathrm{~s}^{-1}$ and $\gamma=1.41 \times 10^{14} \mathrm{~s}^{-1}$. The mode volume calculated with Eq. (9) is mostly real and positive $V=(5-0.4 i) \lambda^{3} / 10^{4}$.

We now evidence the accuracy of the theory with a more complex example where the emission line shape is far from being Lorentzian. We consider a gap antenna made of two closely placed gold nanorods $[4,28]$ and calculate the total SD rate $\Gamma$ for an on-axis dipole located in the center of the gap; see Fig. 3. Fully vectorial calculations show a single asymmetric resonance (circles), whereas, in this spectral range, two QNMs are spatially matched with the dipole; see Fig. 3(a). With the present formalism, $\Gamma$ is given by the sum of the independent contributions $\Gamma_{1}$ and $\Gamma_{2}$ of the two QNMs, which are calculated with Eq. (8) (solid and dashed curves). The sum (bold red curve) predicts a total SD rate in quantitative agreement with fully vectorial calculations. We emphasize that the contribution $\Gamma_{1}$ to the total decay is mostly negative, even if the corresponding mode is spectrally and spatially matched with the dipole. This effect related to energy dissipation is accurately predicted by the present theory; it is formalized by a complex mode volume (a)

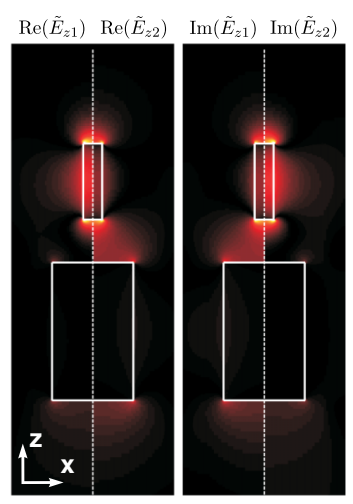

(b)

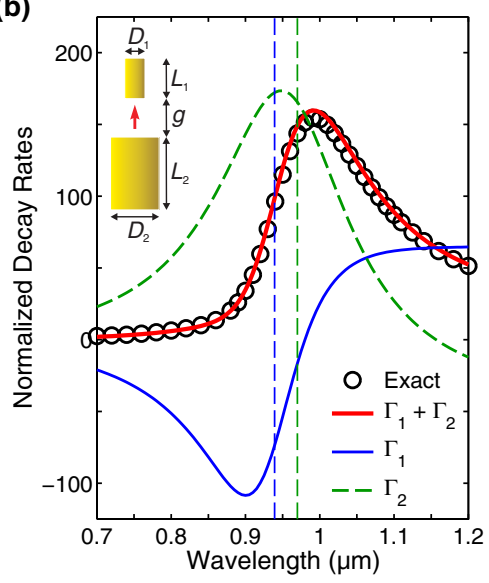

FIG. 3 (color online). Metallic gap resonator. Two gold cylinders (diameters $D_{1}=20 \mathrm{~nm}$ and $D_{2}=85 \mathrm{~nm}$, lengths $L_{1}=80 \mathrm{~nm}$ and $L_{2}=145 \mathrm{~nm}$ ) are separated by a small gap $g=45 \mathrm{~nm}$ and embedded in a host medium with $n=1.5$. (a) Electric field distributions of the two dominant QNMs with complex frequencies $2 \pi c / \tilde{\omega}_{1}=933+77 i \mathrm{~nm}$ and $2 \pi c / \tilde{\omega}_{2}=$ $958+108 i \mathrm{~nm}$. The left (right) panel compares the real (imaginary) parts of $\tilde{E}_{z 1}$ and $\tilde{E}_{z 2}$. (b) Normalized decay-rate spectrum for an on-axis $z$-oriented dipole located in the center of the gap (red arrow). The vertical dashed lines represent $\operatorname{Re}\left(\tilde{\omega}_{1}\right)$ and $\operatorname{Re}\left(\tilde{\omega}_{2}\right)$. The independent contributions $\Gamma_{1}$ and $\Gamma_{2}$ of the two modes are calculated with Eq. (8) and shown by the solid blue and dashed green curves. Their sum (bold red curve) predicts a total decay rate in quantitative agreement with fully vectorial calculations (black circles). 
$V_{1}=(-3-7 i) \lambda^{3} / 10^{4}$. In contrast, the contribution $\Gamma_{2}$ is quasi-Lorentzian with $V_{2}=(4+3 i) \lambda^{3} / 10^{4}$.

In conclusion, we have revisited the usual Purcell factor by providing a self-consistent electromagnetic treatment of the local density of states of dissipative nanoresonators. The latter possess QNMs with a finite lifetime that are not orthogonal in the sense of the energy. Consequently, the contribution of a QNM may decelerate the total decay, even if it is spectrally and spatially matched with the source. This unexpected effect is due to the presence of dissipation and is formalized by the signed term $\operatorname{Re}(1 / V)$ in the generalized Purcell factor of Eq. (10). Moreover, for emitter frequencies detuned from the cavity resonance, the response of the system can be non-Lorentzian. This second effect is taken into account by affecting a complex value to the mode volume; see Eq. (8). This choice may appear to be motivated by mathematical rather than physical considerations, but we emphasize that the new definition of $V$ is fully consistent with the usual one in Eq. (2) that is valid in the limit $Q \rightarrow \infty$. The present theory is a powerful tool since it provides highly accurate semianalytical predictions for most ultrasmall resonators of current interest in nanophotonics, including situations with radiation leakage, absorption, and dispersion. Once a few dominant modes have been calculated, any variation of the dipole frequency, location, or orientation is treated analytically, in contrast to full numerical methods. We therefore believe that the present theory may be useful to engineer further quantum effects, such as strong coupling in weakly damped cavities [29], lasing with ultrasmall plasmonic modes [7-9] or disordered systems [30], or superradiance effects in complex media [31]. Furthermore, we expect that the formalism can be carried out of nanophotonics back to classical antenna theory and used to solve the relevant problem of the radiated power enhancement of electrically small antennas in realistic environments [32].

*christophe.sauvan@institutoptique.fr †philippe.lalanne@institutoptique.fr

[1] E. M. Purcell, Phys. Rev. 69, 681 (1946).

[2] E. Betzig and R. J. Chichester, Science 262, 1422 (1993).

[3] T. H. Taminiau, F. D. Stefani, F. B. Segerink, and N. F. van Hulst, Nat. Photonics 2, 234 (2008).

[4] P. Mühlschlegel et al., Science 308, 1607 (2005).

[5] S. Kühn, U. Hakanson, L. Rogobete, and V. Sandoghdar, Phys. Rev. Lett. 97, 017402 (2006).

[6] M. Khajavikhan, A. Simic, M. Katz, J. H. Lee, B. Slutsky, A. Mizrahi, V. Lomakin, and Y. Fainman, Nature (London) 482, 204 (2012).
[7] D. J. Bergman and M. I. Stockman, Phys. Rev. Lett. 90, 027402 (2003).

[8] R. F. Oulton, V. J. Sorger, T. Zentgraf, R.-M. Ma, C. Gladden, L. Dai, G. Bartal, and X. Zhang, Nature (London) 461, 629 (2009).

[9] M. A. Noginov, G. Zhu, A. M. Belgrave, R. Bakker, V. M. Shalaev, E. E. Narimanov, S. Stout, E. Herz, T. Suteewong, and U. Wiesner, Nature (London) 460, 1110 (2009).

[10] I. S. Maksymov, M. Besbes, J. P. Hugonin, J. Yang, A. Beveratos, I. Sagnes, I. Robert-Philip, and P. Lalanne, Phys. Rev. Lett. 105, 180502 (2010).

[11] J. T. Choy, B. J. M. Hausmann, T. M. Babinec, I. Bulu, M. Khan, P. Maletinsky, A. Yacoby, and M. Lončar, Nat. Photonics 5, 738 (2011).

[12] J.-M. Gérard, Top. Appl. Phys. 90, 269 (2003).

[13] R. K. Chang and A.J. Campillo, Optical Processes in Microcavities (World Scientific, London, 1996).

[14] P. T. Leung, S. Y. Liu, and K. Young, Phys. Rev. A 49, 3057 (1994).

[15] P. T. Kristensen, C. van Vlack, and S. Hughes, Opt. Lett. 37, 1649 (2012).

[16] S. A. Maier, Opt. Quantum Electron. 38, 257 (2006).

[17] A. F. Koenderink, Opt. Lett. 35, 4208 (2010).

[18] S. Derom, R. Vincent, A. Bouhelier, and G. Colas des Francs, Europhys. Lett. 98, 47008 (2012).

[19] L. Novotny and N.F. van Hulst, Nat. Photonics 5, 83 (2011); M. Agio, Nanoscale 4, 692 (2012).

[20] P. Lalanne, C. Sauvan, and J.-P. Hugonin, Laser Photonics Rev. 2, 514 (2008).

[21] A. Fang, T. Koschny, and C. M. Soukoulis, Phys. Rev. B 82, 121102(R) (2010).

[22] L. Novotny and B. Hecht, Principles of Nano-optics (Cambridge University Press, Cambridge, England, 2006).

[23] See Supplemental Material at http://link.aps.org/ supplemental/10.1103/PhysRevLett.110.237401 for more details on the derivation of Eq. (5), for technical elements on the mode volume calculation and for additional numerical tests.

[24] L. Novotny, Phys. Rev. Lett. 98, 266802 (2007).

[25] E. Silberstein, P. Lalanne, J.-P. Hugonin, and Q. Cao, J. Opt. Soc. Am. A 18, 2865 (2001).

[26] A. Armaroli, A. Morand, P. Benech, G. Bellanca, and S. Trillo, J. Opt. Soc. Am. A 25, 667 (2008).

[27] E. D. Palik, Handbook of Optical Constants of Solids (Academic, New York, 1985).

[28] A. Mohammadi, V. Sandoghdar, and M. Agio, New J. Phys. 10, 105015 (2008).

[29] J. P. Reithmaier, G. Sęk, A. Löffler, C. Hofmann, S. Kuhn, S. Reitzenstein, L. V. Keldysh, V.D. Kulakovskii, T. L. Reinecke, and A. Forchel, Nature (London) 432, 197 (2004).

[30] J. Andreasen et al., Adv. Opt. Photonics 3, 88 (2011).

[31] T. V. Teperik and A. Degiron, Phys. Rev. Lett. 108, 147401 (2012).

[32] D. Miron, Small Antenna Design (Pergamon, London, 2006). 